UCRL-ID-130046

\title{
A new six degree of freedom position sensor greatly improves flexible manufacturing - but will manufacturers adapt?
}

C. S. Vann

March 2, 1998

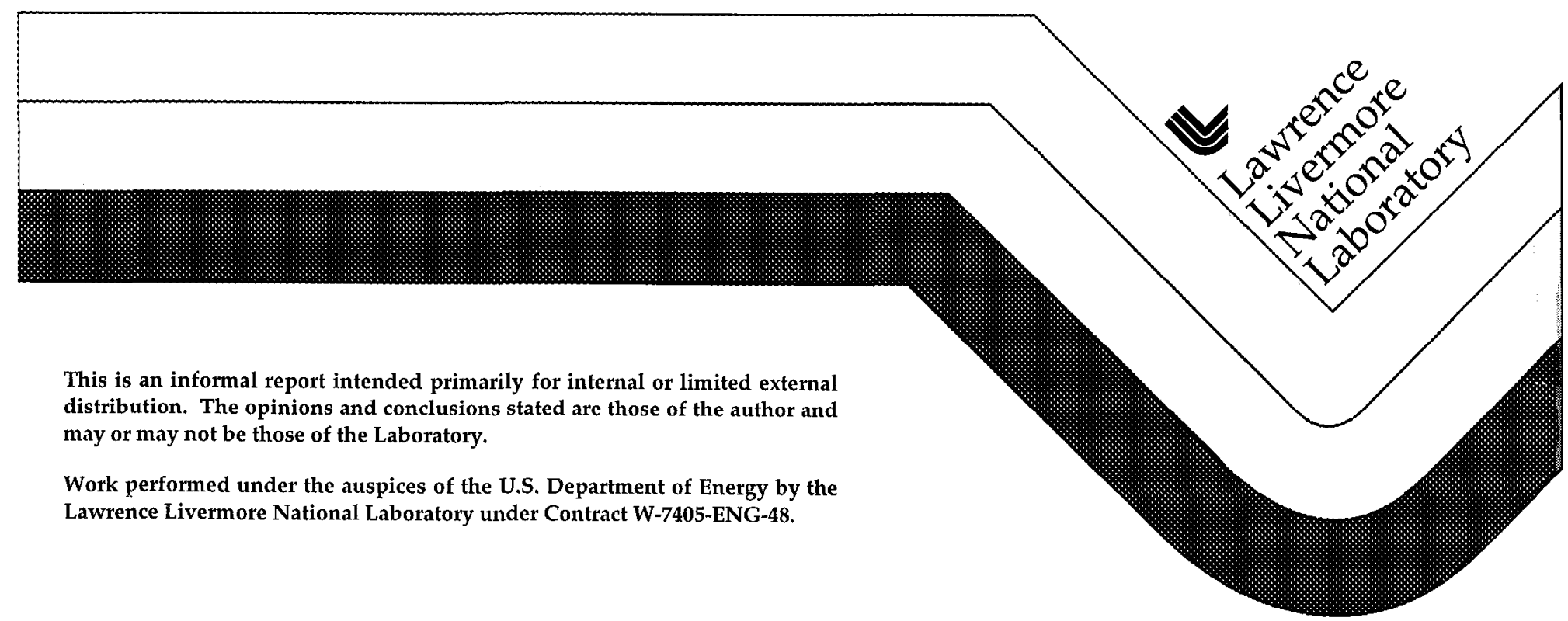




\section{DISCLAIMER}

This document was prepared as an account of work sponsored by an agency of the United States Government. Neither the United States Government nor the University of California nor any of their employees, makes any warranty, express or implied, or assumes any legal liability or responsibility for the accuracy, completeness, or usefulness of any information, apparatus, product, or process disclosed, or represents that its use would not infringe privately owned rights. Reference herein to any specific commercial product, process, or service by trade name, trademark, manufacturer, or otherwise, does not necessarily constitute or imply its endorsement, recommendation, or favoring by the United States Government or the University of California. The views and opinions of authors expressed herein do not necessarily state or reflect those of the United States Government or the University of California, and shall not be used for advertising or product endorsement purposes. 


\title{
VIEWPOINT
}

A new six degree of freedom position sensor greatly improves flexible manufacturing - but will manufacturers adapt?

Charles S. Vann, Ph.D.

\section{The author}

Charles S. Vann is the Deputy Program Leader for the Laser Science and Technology Program, Lawrence Livermore National Laboratory, Livermore, CA 94550, USA, Tel: 510423 8201; Fax 510422 1930; e-mail: vann1@llnl.gov

\begin{abstract}
The manufacturing industry needs better multi-dimensional position sensors to keep pace with increased speed and capabilities of automated equipment and robots. The market for laser and vision sensors is growing at a rapid speed; however, most of the robotmounted sensors are one or two dimensional, three dimensional at most, while the robotics industry has progressed to 6-axis machines, leaving a gross mismatch in its capability to perform and sense the required task. A new laser sensor provides information on up to six degrees of position, enabling robots to sense as well as it can physically manipulate, and opening the door to vast improvements in flexible manufacturing. To expedite these improvements, the manufacturing industry should take a lead and not let an outdated norm hinder implementation.
\end{abstract}

The problem with manufacturing robots today is that they are flexible but unadaptable machines in comparison to humans. Robots have the physical agility to perform many human-like tasks, but even the most advanced manufacturing robots have almost no human-like ability to interpret their environment and make intelligent decisions on their own. Up to now, we have been forced to treat robots as dumb machines, training them to perform each new task, and requiring them to totally rely on exact positioning of the 
robot and part. If a new part or tool is introduced, the robot must be retrained - a major cost of retooling a plant. Worse yet, simple process anomalies such as part misplacement, contamination, wear, slack in the robot arm, or temperature changes can lower product quality, damage robots, stop production lines, or even injure workers. The bottom line is that robots need to be smarter to be more productive.

One main obstacle to smarter robots is their inability to sense their environment as humans do. Humans sense displacement and orientation of objects, i.e. all six degrees of freedom (6-DOF), enabling us to perform complex and intelligent tasks so efforllessly that we take for granted how complex those tasks are for a robot to duplicate. For example, drinking from a can, eating with a fork, or getting dressed are everyday 6-DOF tasks. These seemingly simple tasks for us are complex tasks for robots, and the ability to sense all 6-DOF is absolutely essential to performing them. Exploring our ability even more, humans associate tasks with recognizing an object. For example, if we are given a fork, we know to eat with it rather than drink from it. In other words, we can interpret the task by just seeing the item.

Presently, manufacturing robots are far from having human-like adaptability. Only in some manufacturing situations, do robots have a sensor at all, usually a camera attached to the robot's wrist and providing 2 or 3 dimensional position information. Cameras are sufficient for simple 3-dimensional pick and place operations. However, many robots today have 6-axes, meaning they are physically capable of complex manipulation of parts. For them, sensing only a few of the 6-DOF is insufficient since the robot still must be taught how to manipulate the part in the unsensed axes, or several sensors must be grouped, requiring expensive and complicated software and intermediate reference frames.

A single sensor measuring not only displacement but orientation of an object would be an enabling component for 6-axes robots to adapt to their environment and make decisions with autonomy. At Lawrence Livermore National Laboratory, we have developed and 
tested such a sensor, appropriately called the SixDOF sensor. The SixDOF sensor is noncontact, using a diode laser to illuminate small reflectors on an object or fixture and determining the position of that object from the reflected light and 3 position sensitive detectors inside the sensor. Position is measured in all three translations and all three rotations. The sensor is about the size of a pager and is much faster, less costly, and more accurate then other robot sensors. Furthermore, the reflectors are inexpensive and can be varied in size to code task information, enabling the SixDOF sensor to read from the part which task to perform -- similar to a bar code.

We mounted the SixDOF sensor on a robot in our laboratory and demonstrated its ability to identify the presence of a part, to provide position information in all 6-DOF, and to read a task from the reflectors on the part. Similarly, on a manufacturing line, the SixDOF sensor could read the task and sense its relative position to the part, instructing a robot to align and perform pre-programmed instructions specific to that particular part, e.g. drill holes, weld pieces, etc. - - even if the part were inaccurately positioned and significantly different from the last part. This is possible because part shapes can be exactly defined by 3-dimensional CAD drawings. If the robot knows the task and exactly where it is with respect to the part, then it can work anywhere on the part with precision. Furthermore, with the task assignment coded on the part, a variety of parts can be interchanged in a process without the need to retrain or reprogram the robot. Since the parts are only loosely delivered to the robot, part fixturing doesn't need to be changed either. Tasks which before could only be performed manually or after machine training by a human operator, can now be completed autonomously and with totally flexiblity.

This sounds like the breakthrough that flexible manufacturers have been seeking - a sensor that enables robots to adapt to changing situcations. From my viewpoint, $I$ contend that it is, but from the viewpoint of the manufacturers I have talked with, there is a philosophical issue with implementation. Adding reflectors to parts is another process step; and new steps are costly and must be avoided. In this case, I disagree with this norm for the following two reasons. First, rather than adding a reflective surfacelo a part 
later, it can be made into the part when it is formed, avoiding any cost or delay associated with it. For example, a plastic injection mold with a small, smooth circular area surrounded by a diffuse texture will generate, on every part, the reflective references needed for the SixDOF sensor. Likewise, metals references can be made when a metal part is stamped or forged into its initial shape. Secondly, improvements in efficientcy and adaptablity by referencing to the part rather than to fixed reference frames far outweighs the relatively minor cost of implementation. As described above, aligning each manufacturing component (conveyer belts, fixtures, parts, robots, etc.) to fixed and arbitrary reference frames is wasteful, requiring exact positioning, robot training, and expensive fixtures to keep the process from breaking down. In contrast, with references on the part, sensors like the SixDOF enable robots to adapt to loosely aligned parts without training or expensive fixtures. Furthermore, one set of references can consistently guide many different robots through every process that part undergoes such as milling, welding, inspection, inventory, etc.

It is interesting that the argument against applying reflectors could have also been made against applying bar codes on products. Notably, it is difficult to find a product today without a bar code. Like the bar code and reader, indications are that reflectors on parts and the SixDOF sensor have compelling benefits which could greatly improves flexible manufacturing - but will manufacturers adapt.?

This work was performed under the auspices of the U.S. Department of Energy by Lawrence Livermore National Laboratory under contract Number W-7405ENG-48. 


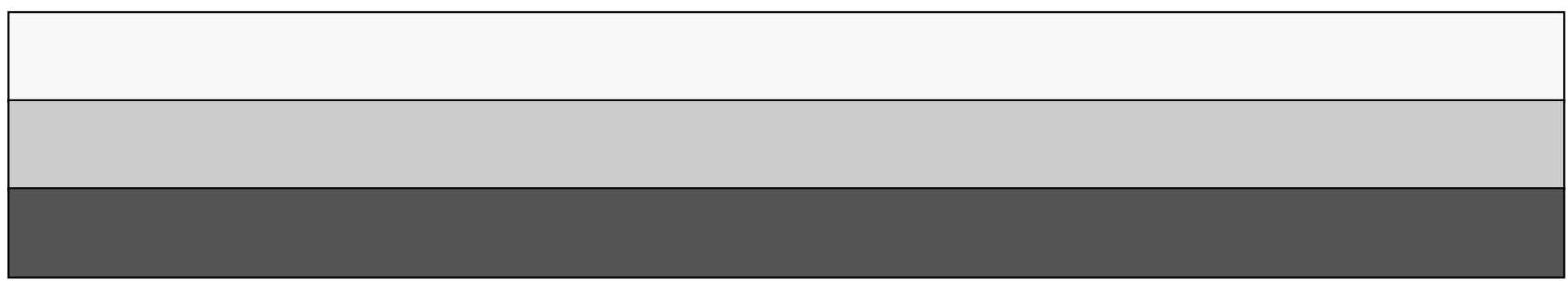

